\title{
Optimal Time-Frequency Distribution Selection for LPI Radar Pulse Classification
}

\author{
Ben Willetts, Matthew Ritchie and Hugh Griffiths \\ Dept. Electronic and Electrical Engineering \\ University College London \\ London, UK \\ [b.willetts, m.ritchie, h.griffiths]@ucl.ac.uk
}

\begin{abstract}
The work presented in this paper shows the performance of various time-frequency distributions when gathering ELectronic INTelligence (ELINT) from an electromagnetic environment that contains transmissions from radars operating in a Low Probability of Interception (LPI) mode. A radar device varying waveform parameters on a pulse-by-pulse basis to enhance sensing capabilities and/or to avoid interception warrants a method that can assign a unique Pulse Descriptor Word (PDW) to each pulse detected. The simulations presented here makes use of a Deep Learning classifier that is fed by timefrequency images of noisy LFM pulses that each have unique signal parameters. The performance of the radar pulse classifier is conveyed for multiple time-frequency methods. The results show that the time-frequency representation requirements for accurate PDW generation varies for each signal parameter being estimated whilst also having a dependence on the SNR of the intercepted signal.
\end{abstract}

Keywords-ELINT, Waveform Classification, Deep Learning, Time-frequency analysis, LPI waveforms

\section{INTRODUCTION}

The modern-day electromagnetic (EM) environment is likely to be heavily cluttered with communications and radar signals particularly in urban areas and along trade/travel routes. The EM environment will contain different types of radar systems that are each designed to observe a particular region in space (as shown in Fig. 1). These systems may have the ability to operate in different modes and hence utilize a variety of waveforms. A radar system changing its mode of operation may be preassigned behavior or due to external influences. Parts of the world in which there is conflict are expected to have a larger presence of radar and jamming signals due to the importance of full spectrum situation awareness in the modern battlefield [1]. LPI waveforms are radar signals that are designed to be able to sense the surrounding environment whilst not giving other radar systems the ability to detect the signal. This creates a game of cat-and-mouse involving LPI radar system designers and those developing ELINT receivers.

The generic Linearly Frequency Modulated (LFM) waveform is still widely used today for radar systems operating in an LPI mode [2] due to its simplicity and effectiveness [3]. As LFM is still commonly used by radar designers, a means of accurately classifying LFM transmissions will need to be robust to other interfering chirp signals. Radars aiming to avoid interception may look to employ elaborate techniques that vary the parameters of the LFM waveform on a pulse-by-pulse basis [4]. A means of extracting the parameters of each LFM pulse accurately will allow the existence of multiple emitters to be detected. A simple LFM signal model will be described in Section II alongside a Pulse Descriptor Word (PDW) which is used to assign a label to each LFM pulse.

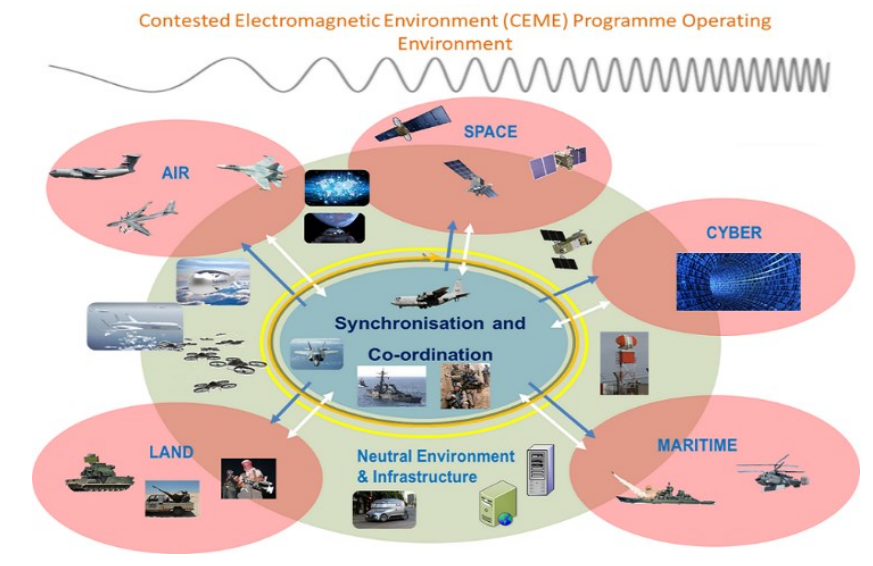

Fig. 1. Different types of radar systems within a modern EM environment [1]

The Time-Frequency (TF) domain can be used to analyze a signal's spectrum over time. It has been widely used to estimate the parameters of a detected signal such as the modulation scheme [5] [6]. Estimating radar signal parameters will allow the sensing capability of its emitter to be estimated. Various TF transforms have been developed in the past to display a signal in the TF domain and each of these methods has particular properties. Examples of four TF techniques will be described in Section III.

Further considerations have to be made when considering the nature of signals in the TF domain when measuring in realistic scenarios. The work here concerns the analysis of LFM signals on a pulse-by-pulse basis, techniques that are used to split a 
pulse-train into individual pulse are described in [7]. The presence of multipath reflections will cause interleaving pulses to be measured and will need further processing methods to extract the fundamental pulse repetition frequency which is described in [8]. Radar systems making use of scanning antenna beam patterns to perform imaging will also cause the SNR of a signal to vary over multiple pulses with respect to an ELINT gathering receiver [9]. The effect of an intercepted emitter using antenna beam scanning techniques on the amplitude modulation of a captured signal is not considered here but can be extracted using the SNR element of the PDW generator described here.

\section{SIGNAL GENERATION \& ESTIMATION}

\section{A. Linear Frequency Modulation}

An ELINT surveillance receiver is required to be able to intercept and classify LPI signals [4]. A simple, deterministic example of an LPI waveform that only requires a relatively inexpensive transceiver device to utilize is the LFM waveform. The complex representation of a baseband LFM pulse with amplitude $A$, Chirp bandwidth $\Delta f$, and Chirp duration $\Delta T$ that is contaminated with uncorrelated AWGN with zero-mean and a variance of $\sigma_{n}$ is given by (1).

$$
s(t)=A e^{j\left(2 \pi\left(\frac{\Delta f}{\Delta T}\right) t^{2}+\varphi\right)}+A W G N\left(\mu_{n}=0, \sigma_{n}\right)
$$

$$
\text { for } 0<t<\Delta T
$$

It is assumed that the In-phase and Quadrature components are measured with independent AWGN. A complex measurement of the waveform will enhance the signal analysis due to the phase information being included.

\section{B. Pulse Descriptor Words}

A PDW is an array that consists of estimated signal parameters in an attempt to gather ELINT of the electromagnetic environment [7]. A PDW may be constructed to be versatile or can be made waveform-specific.

A radar designer will select a waveform to meet a specification that typically details a systems capability in measuring the range, angle and velocity of an object. A passive receiver gaining ELINT will need to estimate these waveform parameters in the presence of other signals and noise. A radar designer can employ waveform design techniques to improve the capability of a radar system whilst also making the transmissions robust to interception/interrogation. Agile waveforms that vary waveform parameters on a pulse-by-pulse basis to achieve these objectives will need to be considered.

The PDW considered here for noisy LFM pulses will consist of three elements: chirp duration, chirp bandwidth and SNR (2). The PDW array has three elements for the simulations presented here although this could be modified to suite more elaborate waveforms or even for emitter identification by instead estimating signal imperfections rather than signal parameters.

$$
P D W=\left[\Delta f_{e}, \Delta T_{e}, S N R_{e}\right]
$$

The PRF (Pulse Repetition Frequency) is not estimated due to this parameter usually being processed at different stages of an ELINT gathering system as conveyed in [7] and [10].

\section{TIME-FREQUENCY REPRESENTATION}

\section{A. Cohen's Class}

Cohen's class [11] describes a set of bilinear distributions that allow time-frequency analysis of complex/real signals and is represented by (3) [12]. A distribution that is a member of the Cohen's class can be evaluated on a single signal in which (3) contains the autocorrelation function or it can be used to crosscorrelate between two signals.

$$
C(t, f)=\int s\left(\theta+\frac{\tau}{2}\right) s^{*}\left(\theta-\frac{\tau}{2}\right) \phi(t-\theta, \tau) e^{-j 2 \pi f \tau} d \theta d \tau
$$

The two-dimensional kernel function $\Phi$ in (4) allows filtering in the TF domain and is given by the Fourier transform of $\phi$ in (3):

$$
\Phi(\theta, \tau)=\mathcal{F}(\phi(\mathrm{t}, \tau))
$$

Cross-term products that occur for signals with multiple frequency components can degrade signal parameter extraction and thus the kernel function allows the suppression of this form of interference.

A unique kernel function will produce a distinct auto-term signature and cross-term interference pattern. The finer the resolution of the TF signature, the higher the SNR for a given noisy signal. Cross-term filtering can make signal parameter extraction more accurate and less computationally extensive.

\section{B. Examples of Time-Frequency Distributions}

The Short Time Frequency Transform (STFT) or spectrogram method is the TF given by the kernel function in (5).

$$
\Phi_{G}(\theta, \tau)=\int h^{*}\left(u-\frac{\tau}{2}\right) h\left(u+\frac{\tau}{2}\right) e^{-j \theta u} d u
$$

The STFT is referred to as the Gabor transform (GT) when a Gaussian window is used. The STFT is limited by the resolution of the window imposed by the windowing function $h(\cdot)$. This limitation can be exceeded by using alternative TF distributions.

The Wigner-Ville Distribution (WVD) is the Fourier Transform of the autocorrelation function which is the Cohen class expression in (3) with a unity kernel function (6). The finest TF resolution is achieved with the WVD but at the consequence of suffering from severe cross-term interference patterns for signals with multiple-frequency components.

$$
\Phi_{W V}(\theta, \tau)=1
$$

The cross-term interference can be suppressed by applying a Gaussian smoothening function with the expense of a loss in resolution. The WVD with a Gaussian smoothening function applied is commonly referred to as the Pseudo Wigner-Ville distribution (PWVD).

The Choi-Williams distribution (CWD) [13] has a kernel function that gives it a better resolution than the Gabor 
Transform and less cross-term interference than the WVD. The CWD method is computed with (3) whilst using the kernel function in (7). The parameter $\alpha$ gives variability in the axis in which the TF filtering is applied.

$$
\Phi_{C W}(\theta, \tau)=e^{-\alpha(\theta \tau)^{2}}
$$

Equations (3) to (7) are in continuous form but will need to be implemented for discrete signals due to the stochastic component of a noisy LFM pulse (1). The Discrete TF codes made available at [14] and [15] will be used to perform all of the time-frequency results presented here. The four time-frequency distribution/transforms of interest here have been previously labeled in bold.

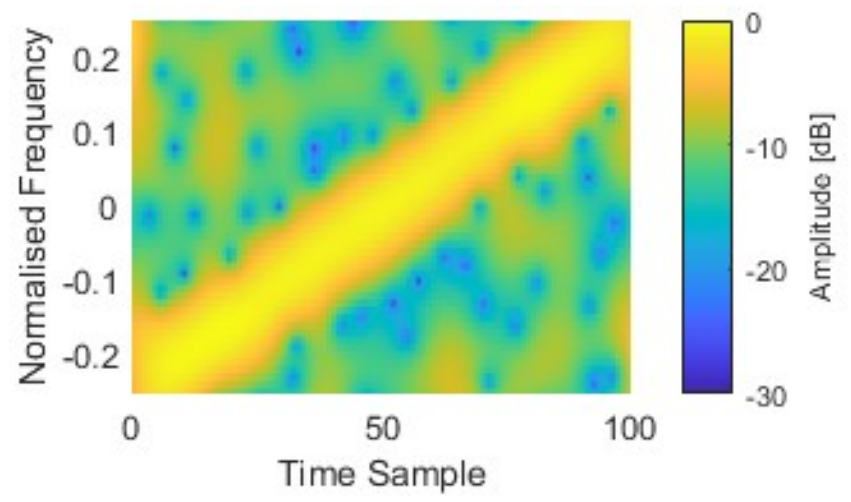

a)

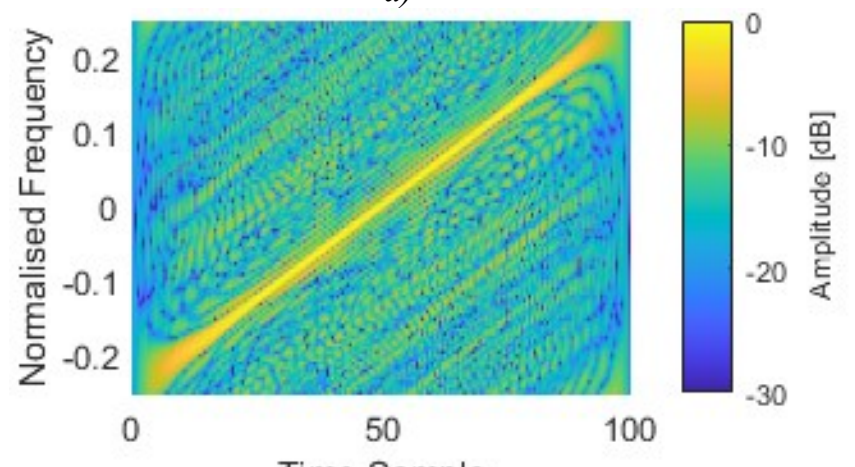

Time Sample

b)

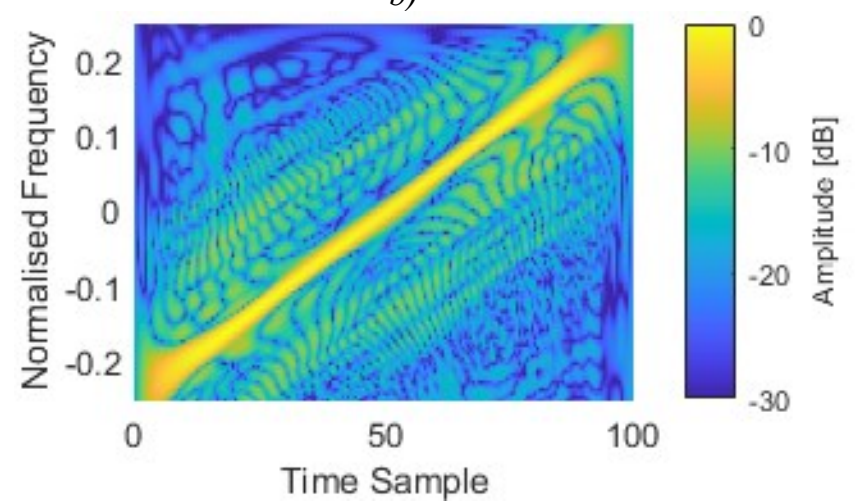

c)

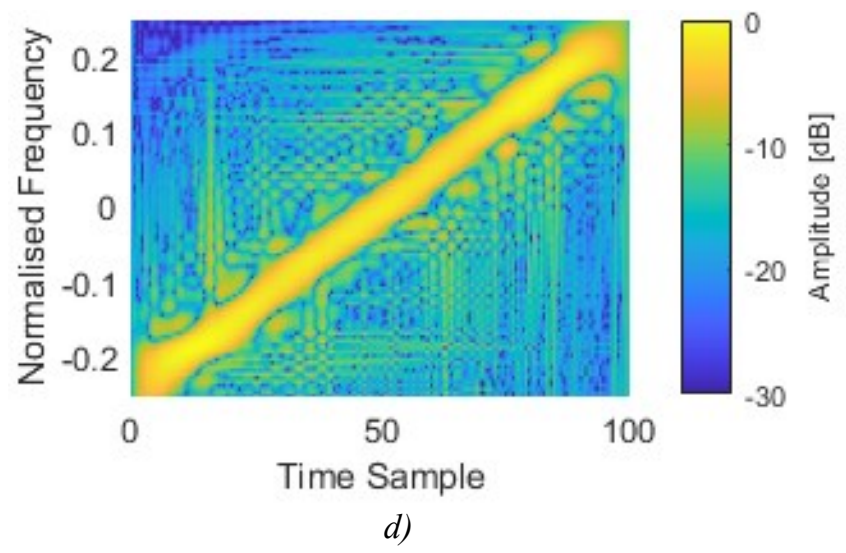

Fig. 2. Noisy LFM data ( $\mathrm{SNR}=10 \mathrm{~dB}, \Delta f=0.5 F s$ and $\Delta T=100 / F s$ ) processed using different TF methods: (a) GT (b) WVD (c) PWVD (d) CWD

The baseband LFM pulses shown in Fig. 2 are of the same noisy signal although each of the images have been produced by a different TF technique. These plots illustrate the unique TF resolutions and interference patterns produced by each $\mathrm{TF}$ distribution.

\section{DEEP LEARNING CLASSIFICATION METHOD}

The classification method used in the work presented here is based on AlexNet [16] which is a Convolutional Neural Network (CNN) developed for image classification. An article was previously published that makes use of the same Deep Learning method but instead for the classification of modulation schemes [5]. Each CNN input image was generated by inserting a noisy LFM pulse into each of the four time-frequency distributions stated in Section III. The image generated by each $\mathrm{TF}$ transform was down-sampled to $240 \times 240$ pixels for consistency. RGB values were then extracted for each generated image and these color values correspond to the magnitude of the $\mathrm{TF}$ transform output. The overall signal generation and classification procedure is visible in Fig. 3 and the $\mathrm{CNN}$ training parameters used are displayed in Table I.

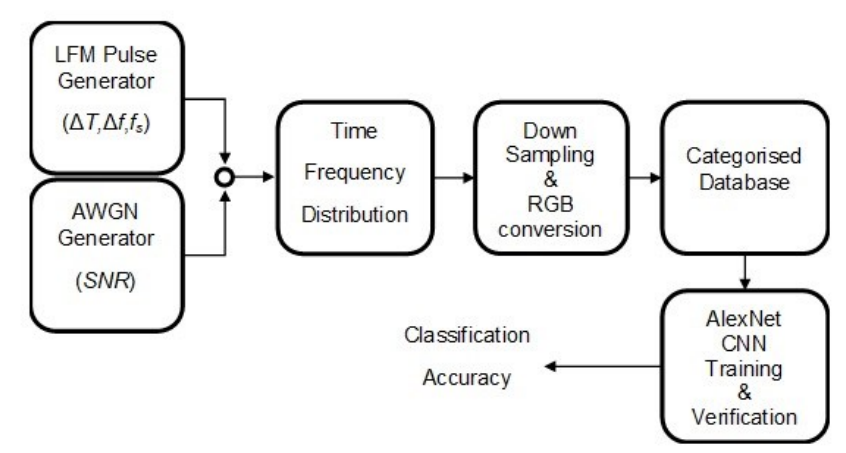

Fig. 3. Block diagram showing the simulation process for each PDW element 
TABLE I. DEEP LEARNING PARAMETERS USED

\begin{tabular}{|l|l|}
\hline \multicolumn{1}{|c|}{ Parameter } & Value \\
\hline Maximum Epochs & 5 \\
\hline Initial Learn Rate & 0.001 \\
\hline Weight Learn Rate Factor & 20 \\
\hline Bias Learn Rate Factor & 20 \\
\hline
\end{tabular}

To assign a PDW to a measured signal, a set of categories were defined prior to the training of the $\mathrm{CNN}$. The following uniformly sampled categories were constructed for each of the three PDW elements:

$$
\begin{aligned}
& C_{\Delta f e}=\left[\frac{F s}{10}, 2 \frac{F s}{10}, \ldots, 5 \frac{F s}{10}\right] \\
& C_{\Delta T e}=[10 T s, 20 T s, \ldots, 100 T s] \\
& C_{S N R e}=[-9 d B,-6 d B \ldots, 9 d B]
\end{aligned}
$$

$T s$ and $F s$ are the sampling time and frequency respectively. The category increments of $F_{s} / 10,10 T_{s}$ and 3 decibels in (8), (9) and (10) respectively were arbitrary chosen but deemed acceptable to separate repeating LFM with significantly different sensing capabilities. The work presented here will not consider sub-Nyquist sampling of signals. Each $\mathrm{CNN}$ had a dataset of 1000 noisy LFM signals (1) that were generated with signal parameters generated by uniform pdfs. The uniform pdfs of the three signal parameters had ranges which correspond to the max and min values in the category vectors (8), (9) and (10). The TF representation of each noisy LFM signal was then stored in an image format inside a database alongside the corresponding signal parameter used for generation $(\Delta F, \Delta T$ and $S N R$ ). The CNN is trained with $80 \%$ of the dataset and then tested on with $10 \%$ of the dataset. The remaining $10 \%$ was then used for validation.

\section{RESULTS \& ANALYSIS}

\section{A. $\Delta f_{e} \& \Delta T_{e}$ classification using pulses with constant $S N R$}

The simulation results presented here show the optimal TF method for estimating the chirp period and bandwidth elements of (2). The CNN training database made use of noisy LFM pulses that have been generated with the same SNR value and this was done iteratively for each value in (10). The classification accuracy incurred for different SNR values are shown in Fig. 4. Table II shows the optimal TF method alongside the achieved maximum classification accuracy for all of the TF methods used. The LFM bandwidth estimation accuracy improved by $60 \%$ over the SNR range simulated whereas the pulse period estimation accuracy stayed approximately constant at around $80 \%$. The WVD distribution was found to be the optimal TF distribution for all of the cases simulated apart from the $S N R=3 \mathrm{~dB}$ and $S N R=-3 \mathrm{~dB}$ scenarios when classifying the pulse period. A more accurate signal parameter extractor might be expected when using TF methods that filter out cross-term interference (all but WVD) for high SNR scenarios $(S N R>0 \mathrm{~dB})$ although for the Deep Learning classifier used here this was not the case. Using the kernel function in (3) to filter out cross-term interference can reduce the capability of a CNN to extract sufficient features for correct classification.

TABLE II. OPTIMAL TFD AND MAXIMUM CLASSIFICATION ACCURACY

\begin{tabular}{|c|c|c|c|}
\hline $\mathrm{SNR}$ & $\Delta f_{e}$ & $\Delta T_{e}$ & $\left(\Delta f_{e},+\Delta T_{e}\right) / 2$ \\
\hline$-9 \mathrm{~dB}$ & $28 \%$ & $78 \%$ & $53 \%$ \\
\hline$-6 \mathrm{~dB}$ & $52 \%$ & $82 \%$ & $67 \%$ \\
\hline$-3 \mathrm{~dB}$ & $72 \%$ & $81 \%$ & $76 \%$ \\
\hline $0 \mathrm{~dB}$ & $81 \%$ & $82 \%$ & $81 \%$ \\
\hline $3 \mathrm{~dB}$ & $86 \%$ & $83 \%$ & $83 \%$ \\
\hline $6 \mathrm{~dB}$ & $84 \%$ & $83 \%$ & $84 \%$ \\
\hline $9 \mathrm{~dB}$ & $88 \%$ & $81 \%$ & $85 \%$ \\
\hline
\end{tabular}

TABLE III. CLASSIFICATION ACCURACY OF PDW (2) FOR EACH TFD

\begin{tabular}{|l|c|c|c|c|}
\hline & $\Delta f_{e}$ & $\Delta T_{e}$ & $\mathbf{S N R}_{\mathrm{e}}$ & $\left(\Delta f_{e},+\Delta T_{e}+S_{\left.N R_{e}\right) / 3}\right.$ \\
\hline$\underline{\mathbf{G T}}$ & 79 & 68 & $\mathbf{8 0}$ & 76 \\
\hline$\underline{\text { WVD }}$ & 84 & $\mathbf{8 7}$ & 79 & $\mathbf{8 3}$ \\
\hline$\underline{\mathbf{P W V D}}$ & $\mathbf{8 6}$ & 82 & 75 & 81 \\
\hline$\underline{\mathbf{C W D}}$ & 82 & 83 & 77 & 81 \\
\hline
\end{tabular}

Optimal TFD color scheme

\begin{tabular}{|c|c|}
\hline GT & PWVD \\
\hline WVD & CWD \\
\hline
\end{tabular}



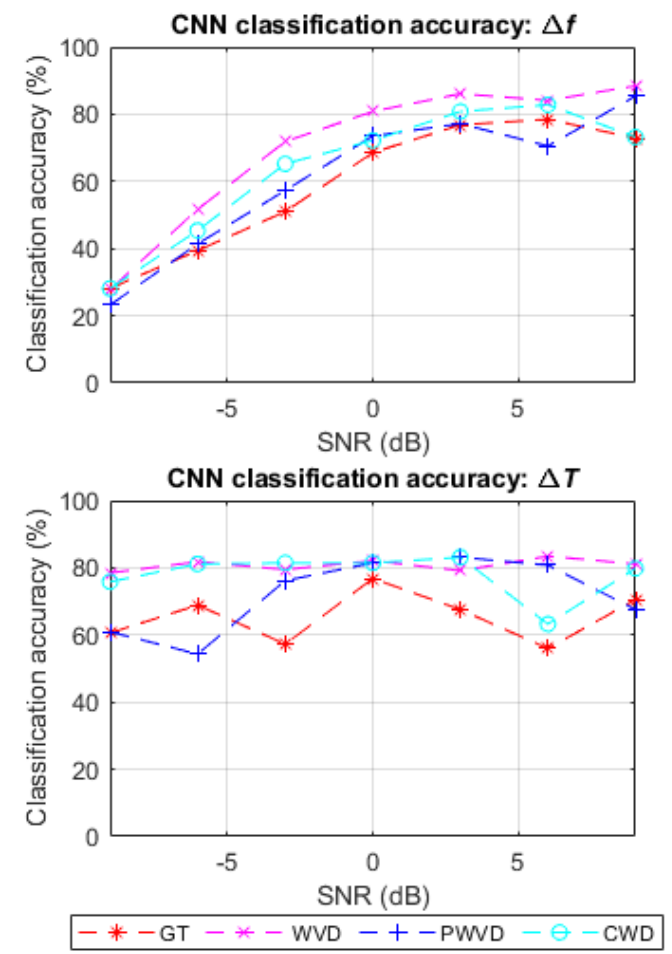

Fig. 4. Classification accuracy for signals with different signal-to-noise ratios

\section{B. $\Delta f_{e}, \Delta T_{e} \& S N R_{e}$ classification with categories in (8)-(10)}

Table III displays the classification accuracy when using a database of 1000 noisy LFM pulses that were generating using the parameters in (8), (9) and (10). Training a CNN to classify each element of (2) for SNR values between $-9 \mathrm{~dB}$ and $9 \mathrm{~dB}$ showed a different optimal TF distribution for each of the three PDW elements. This shows that training the CNN with data containing a larger range of noise statistics alters the CNN operation significantly. The Wigner-Ville distribution was seen to have the highest classification accuracy overall. The results show that using multiple $\mathrm{TF}$ distributions to classify different elements of a PDW can lead to a more accurate classifier compared to using a single TF method.

\section{CONCLUSION}

The importance of gaining as much information as possible about the surrounding electromagnetic environment for EM superiority has been stated. The ability to generate pulse descriptor words from noisy LPI radar signals by using a Deep Learning classifier on time-frequency images has been shown. Simulation results presented here have shown that the filtering of cross-term interference by using a kernel function was seen to degrade the performance of a Deep Learning LPI pulse classifier. Results have also been presented that show the use of multiple time-frequency distributions for different elements of a pulse descriptive word can improve the classification accuracy.

\section{FUTURE WORK}

The full version of this paper will extend the work presented here to other waveforms modulation types such as phase-coded and non-linear frequency modulated waveforms. This will involve the generation of more elaborate Pulse Descriptor Words. The classification algorithms robustness to the presence of other sources of noise such as clutter and jamming devices are also of interest to the research.

\section{ACKNOWLEDGMENT}

This work was supported by the University Defence Research Collaboration (UDRC) consortium and was jointly funded by the Engineering and Physical Sciences Research Council (EPSRC) and the Defence Science and Technology Laboratory (Dstl).

\section{REFERENCES}

[1] Defence and Security Accelerator (DASA), "The Invisible Battlespace" GOV.UK. https://www.gov.uk/government/publications/competitionthe-invisible-battlespace/competition-document-the-invisiblebattlespace (accessed Oct. 31, 2019).

[2] Thales Group, "Scout Mk3," 2016. [Online]. Available: https://www.thalesgroup.com/sites/default/files/database/d7/asset/docum ent/scout mk3-v01.pdf

[3] A. G. Stove, "Linear FMCW radar techniques," IEE Proceedings F Radar and Signal Processing, vol. 139, no. 5, pp. 343-350, Oct. 1992.

[4] R. G. Wiley, Electronic Intelligence: the interception of radar signals. Dedham, MA: Artech House, 1985.

[5] MathWorks, "Radar Waveform Classification Using Deep Learning." MathWorks.com. https://uk.mathworks.com/help/phased/examples/mod ulation-classification-of-radar-and-communication-waveforms-usingdeep-learning.html (accessed Oct. 15, 2019).

[6] P. E. Pace, Detecting and Classifying Low Probability of Intercept Radar. Artech House, 2009.

[7] E. Granger et al., "A what-and-where fusion neural network for recognition and tracking of multiple radar emitters," in Neural Networks, vol. 14, no. 3, pp. 325-344, Apr. 2001.

[8] D. J. Milojevic and B. M. Popovic, "Improved algorithm for the deinterleaving of radar pulses," IEE Proceedings F - Radar and Signal Processing, vol. 139, no. 1, pp. 98-104, Feb. 1992.

[9] S. Robertson, Practical ESM analysis. Artech House, 2019.

[10] J. P. Kauppi et al., "Hierarchical classification of dynamically varying radar pulse repetition interval modulation patterns," Neural Networks, 23(10), 1226-1237, Dec. 2010.

[11] L. Cohen, "Time-frequency distributions-a review," Proceedings of the IEEE, vol. 77, no. 7, pp. 941-981, July 1989.

[12] V. C. Chen and H. Ling, Time-Frequency Transforms for Radar Imaging and Signal Analysis. Artech House, 2002.

[13] H. Choi and W. J. Williams, "Improved time-frequency representation of multicomponent signals using exponential kernels," IEEE Transactions on Acoustics, Speech, and Signal Processing, vol. 37, no. 6, pp. 862-871, June 1989.

[14] DiscreteTFD-v1.2. (2013). J. C. O’Neill. Accessed: Oct. 15, 2019. [Online]. Available: http://tfd.sourceforge.net/

[15] Time-Frequency toolbox. (1996). CNRS (France). Accessed: Oct. 17, 2019. [Online]. Available: http://tftb.nongnu.org/

[16] A. Krizhevsky et al., "ImageNet classification with deep convolutional neural networks," in Advances in neural information processing systems, vol. 1, Dec. 2012, pp. 1097-1105. 